\title{
Apigenin Protects Mouse Retina against Oxidative Damage by Regulating the Nrf2 Pathway and Autophagy
}

\author{
Yuanzhong Zhang, ${ }^{1}$ Yan Yang, ${ }^{2}$ Haitao $\mathrm{Yu}^{3}{ }^{\mathrm{Min}} \mathrm{Li},{ }^{4} \mathrm{Li} \mathrm{Hang},{ }^{1}$ and Xinrong Xu $\mathbb{D}^{1}$ \\ ${ }^{1}$ Department of Ophthalmology, Jiangsu Province Hospital of Chinese Medicine (Affiliated Hospital of Nanjing University of \\ Chinese Medicine), Nanjing 210029, China \\ ${ }^{2}$ Department of Ophthalmology, The Second Affiliated Hospital of Nanjing University of Chinese Medicine, Nanjing 210017, China \\ ${ }^{3}$ School of Pharmacy, Nanjing University of Chinese Medicine, Nanjing 210023, China \\ ${ }^{4}$ Department of Ophthalmology, Liyang Branch of Jiangsu Province Hospital of Chinese Medicine, Liyang 213300, China
}

Correspondence should be addressed to Xinrong Xu; 13851641312@qq.com

Received 27 March 2020; Accepted 29 April 2020; Published 14 May 2020

Guest Editor: Francisco Jaime B. Mendonça Junior

Copyright (c) 2020 Yuanzhong Zhang et al. This is an open access article distributed under the Creative Commons Attribution License, which permits unrestricted use, distribution, and reproduction in any medium, provided the original work is properly cited.

\begin{abstract}
Oxidative stress is a critical factor in the pathology of age-related macular degeneration (AMD). Apigenin (AP) is a flavonoid with an outstanding antioxidant activity. We had previously observed that AP protected APRE-19 cells against oxidative injury in vitro. However, AP has poor water and fat solubility, which determines its low oral bioavailability. In this study, we prepared the solid dispersion of apigenin (AP-SD). The solubility and dissolution of AP-SD was significantly better than that of the original drug, so the oral bioavailability in rats was better than that of the original drug. Then, the effects of AP-SD on the retina of a model mouse with dry AMD were assessed by fundus autofluorescence (FAF), optical coherence tomography (OCT), and electron microscopy; the results revealed that AP-SD alleviated retinopathy. Further research found that AP-SD promoted the nuclear translocation of Nrf2 and increased expression levels of the Nrf2 and target genes HO-1 and NQO-1. AP-SD enhanced the activities of SOD and GSH-Px and decreased the levels of ROS and MDA. Furthermore, AP-SD upregulated the expressions of p62 and LC3II in an Nrf2-dependent manner. However, these effects of AP-SD were observed only in the retina of Nrf2 WT mice, not in Nrf2 KO mice. In addition, the therapeutic effect of AP-SD was dose dependent, and AP did not work. In conclusion, AP-SD significantly enhanced the bioavailability of the original drug and reduced retinal oxidative injury in the model mouse of dry AMD in vivo. The results of the underlying mechanism showed that AP-SD upregulated the expression of antioxidant enzymes through the Nrf2 pathway and upregulated autophagy, thus inhibiting retinal oxidative damage. AP-SD may be a potential compound for the treatment of dry AMD.
\end{abstract}

\section{Introduction}

Age-related macular degeneration (AMD) is a leading cause of vision loss in the elderly. Clinically, AMD falls into two categories: dry AMD and wet AMD. The dry AMD presents as retinal pigment proliferation or depigmentation, drusen, and advanced geographic atrophy. The wet AMD is characterized by choroidal neovascularization (CNV), leading to retinal exudation and hemorrhage, and eventually severe visual impairment. In recent years, the treatment of wet AMD has made significant progress due to the widespread use of antivascular endothelial growth factor (VEGF) drugs
[1]. Currently, there are no effective treatment options for dry AMD.

A large number of evidences indicate that oxidative damage of retinal pigment epithelium (RPE) is a main etiology of AMD. The RPE cell has abundant mitochondria and produces a large amount of reactive oxygen species (ROS) in a high-oxygen environment [2]. In addition, one of the major functions of RPE is the phagocytosis and degradation of photoreceptor outer segments (POS). POS is rich in unsaturated fatty acids, and the part being not degraded by RPE forms lipofuscin, which increases ROS generation while exposed to light [3]. The nuclear factor E2-related factor 2 (Nrf2) 
pathway is a primary system employed by the RPE to neutralize oxidative stress and maintain cellular homeostasis [4]. In a physiological condition, Nrf2 is constitutively targeted for degradation by the multisubunit, E3 ubiquitin lipase KEAP1. Oxidative stress dissociates KEAP1 and stabilizes Nrf2. The transcription factor rapidly translocates to the nucleus, heterodimerizes with Maf proteins, and binds to the antioxidant response elements (AREs) in the promoters of its cognate target genes, inducing the expression of genes encoding heme oxygenase-1 (HO-1), quinone oxidoreductase-1 (NQO-1), glutathione peroxidase (GSH-Px), superoxide dismutase (SOD), and catalase (CAT). These enzymes can quickly scavenge ROS and protect the body from injuries caused by active substances or toxic substances [5]. Thus, the activation of the Nrf2 pathway could reduce the oxidative damage of RPE, suggesting that the Nrf2 pathway is a potential target for AMD treatment [6].

As Nrf2 pathway does, autophagy keeps cellular homeostasis in situations of oxidative stress; therefore, its failure is associated with aging and many diseases, such as cancer, cardiovascular diseases, and neurodegenerative diseases [7]. The impairment of autophagy has been shown to be one cause of the development and progression of AMD [8]. Impaired autophagy significantly reduced the degradation of POS, leading to a deposit of undegraded POS under RPE, and finally an increase of ROS generation $[9,10]$. The autophagy is increased and the Nrf2 pathway is activated at the same time while the cell is in oxidative stress. Polyunsaturated fatty acids rapidly increase the generation of ROS in RPE and upregulate the expression of Nrf2 and SQSTM1/p62 proteins [11]. Studies have shown that many inducers of the Nrf2 pathway can also induce autophagy [12-14]. Correspondingly, a deficiency of Nrf2 reduces SQSTM1/p62 expression [15]. Clearly, there is a strong correlation between autophagy and Nrf2 pathways, which makes sense for AMD.

Apigenin (AP) is a bioactive flavonoid which is obtained from several fruits and vegetables. AP has been reported to exhibit antioxidant, anti-inflammatory, and anticancer activities $[16,17]$. Our previous study has shown that AP exhibited protective effects on ARPE-19 cells against tert-butyl hydroperoxide- (t-BHP-) induced oxidative injury, which were associated with its antioxidant properties dependent upon the activation of Nrf2 signaling [18]. However, AP has the disadvantages of poor water and fat solubility and low bioavailability. Studies have indicated that the preparation of solid dispersion could significantly increase the solubility and dissolution rate of drugs, which can further enhance the bioavailability of drugs $[19,20]$. In the current study, we prepared the solid dispersion of apigenin (AP$\mathrm{SD})$, determined its bioavailability in rats, and evaluated its protective effects on retinal injury induced by oxidative stress in Nrf2 WT and Nrf2 KO mice.

\section{Materials and Methods}

2.1. Chemicals, Reagents, and Antibodies. Apigenin was from Sigma Chemical (St. Louis, MO, USA). Polyvinylpyrrolidone (PVP K30) was from J\&K Chemical Ltd. (Shanghai, China). Hydroquinone (HQ) was from Alfa Aesar (Heysham, Lanca- shire, UK). The primary antibodies used in western blot were obtained from the following source: Nrf2, HO-1, NQO-1, p62, GAPDH, and Lamin B (Abcam, UK) and LC3 (Cell Signaling Technology, USA); the secondary antibody Goat AntiRabbit IgG/HRP was from (Abcam, UK).

2.2. Preparation of AP-SD. With the orthogonal experiment, we assigned PVP K30 as the carrier to prepare the solid dispersion of $\mathrm{AP}$, the mass ratio of $\mathrm{AP}$ and $\mathrm{PVK} \mathrm{V} 30$ is 1: 9, and anhydrous ethanol is used as the reaction solvent to prepare AP-SD. Briefly, AP and PVK V30 at a mass ratio of 1:9 were dissolved in absolute ethanol of adequate volume with full mixing. After the mixture was evaporated in vacuo and vacuum drying at $50^{\circ} \mathrm{C}$, the $\mathrm{AP}-\mathrm{SD}$ was obtained for experiments.

We then determined the standard curve of AP-SD. The chromatographic conditions were Waters $600 \mathrm{C}_{18}$ column, methanol-0.1\% $\mathrm{CH}_{3} \mathrm{COONa}$ solution $(v / v 60: 40)$ as the mobile phase, $337 \mathrm{~nm}$ wavelength, $1.0 \mathrm{~mL} / \mathrm{min}$ flow velocity, $40^{\circ} \mathrm{C}$ column temperature, and $10 \mu \mathrm{L}$ sample size. The control solution was prepared according to following the procedures. AP of $10 \mathrm{mg}$ was dissolved in ethanol and volumed in $50 \mathrm{~mL}$ volumetric flask, and thereby the AP control solution at a concentration of $1 \mathrm{mg} / \mathrm{mL}$ was obtained. Anhydrous ethanol was used to dilute the control solution to yield a series of solutions at $1,2,4,8,16$, and $32 \mu \mathrm{g} / \mathrm{mL}$. Each solution of $10 \mu \mathrm{L}$ was injected into a high-performance liquid chromatograph (HPLC, Waters 600, USA) for measurement of the peak area. Concentrations of AP $([C])$ were marked at the horizontal ordinate and peak areas $(A)$ at the longitudinal coordinate giving the standard curve equation: $A=32786.6$ $\times[C]-160.4(R=0.9991)$, linear range $0.56-32.7 \mu \mathrm{g} / \mathrm{mL}$.

2.3. Determination of Solubility. AP and AP-SD (equivalent to $10 \mathrm{mg}$ of $\mathrm{AP}$ ) were precisely weighted and added into $100 \mathrm{~mL}$ conical flask, and then, distilled water/chloroform of $20 \mathrm{~mL}$ was added. The solutions were incubated in a $25^{\circ} \mathrm{C}$ thermostatic oscillator for $24 \mathrm{hr}$. Each sample of $5 \mathrm{~mL}$ was filtered through a $0.45 \mu \mathrm{m}$ microporous membrane. The successive filtrate of $10 \mu \mathrm{L}$ was subjected to HPLC analyses. Control solution of $10 \mu \mathrm{L}$ at $8 \mu \mathrm{g} / \mathrm{mL}$ was spontaneously analyzed by HPLC. The equilibrium solubility of both samples in water and chloroform was measured, respectively, based on the peak area.

2.4. Determination of Dissolution Rate. AP and AP-SD (both containing $10 \mathrm{mg}$ pure AP) were evenly dispersed in the dissolution medium (phosphate buffer saline, $\mathrm{pH} 7.4,37^{\circ} \mathrm{C}, \mathrm{V}$ $=900 \mathrm{~mL}$ ) and centrifuged at $100 \mathrm{r} / \mathrm{min}$. Each sample of $5 \mathrm{~mL}$ was collected at the time points of $5,10,15,20,30$, 45,60 , and $90 \mathrm{~min}$, respectively, and subjected to filtration. The successive filtrate of $1 \mathrm{~mL}$ was volumed in $10 \mathrm{~mL}$ volumetric flask with distilled water. HPLC analyses were used, and mass concentrations were calculated through introducing a peak area into the standard curve equation followed by calculation of the cumulative dissolution percentage.

2.5. Determination of Plasma Concentrations of AP. Twelve Sprague Dawley rats (Model Animal Research Centre of Nanjing University (Nanjing, China) were randomly divided 
into two groups: the AP and AP-SD groups $(n=6)$. Rats of both groups were intragastrically administrated with corresponding drugs containing pure AP at $50 \mathrm{mg} / \mathrm{kg}$. Blood samples were collected from orbit at time points of $0.25,0.5,1,2$, $4,8,12$, and $24 \mathrm{hr}$ of each rat. After adding heparin, blood samples were centrifuged at $3000 \mathrm{r} / \mathrm{min}$ for $15 \mathrm{~min}$, and the supernatants were collected for examination. $200 \mu \mathrm{L}$ plasma was mixed with $600 \mu \mathrm{L}$ of silybin solution (internal standard, $13.3 \mu \mathrm{g} / \mathrm{mL}$ in methanol) and vortex mixed for $5 \mathrm{~min}$. After centrifugation for $5 \mathrm{~min}$ at $10000 \mathrm{r} / \mathrm{min}$, the supernatant was collected. The rest of the residue was extracted with another $300 \mu \mathrm{L}$ of methanol by vortex mixing for $5 \mathrm{~min}$ and centrifuged for $5 \mathrm{~min}$ at $10000 \mathrm{r} / \mathrm{min}$ again, and the obtained supernatant was pooled together with the previous supernatant. Then, the total supernatant was dried under a nitrogen flow at $35^{\circ} \mathrm{C}$ and the obtained residues were reconstituted with $200 \mu \mathrm{L}$ of methanol. After centrifugation for another $5 \mathrm{~min}$ at $10000 \mathrm{r} / \mathrm{min}$, an aliquot of $20 \mu \mathrm{L}$ supernatant was analyzed by HPLC for determining drug concentrations. Maximum plasma concentration $\left(C_{\max }\right)$ and time to reach maximum concentration $\left(T_{\max }\right)$ were obtained directly from the concentration-time curve; area under the plasma concentration-time curve from zero to the time of the final sample measurement $\left(\mathrm{AUC}_{0-24}\right)$ was calculated using the statistical software GraphPad Prism 5 (GraphPad Software Inc., CA, USA).

2.6. Animal Experimental Procedures. C57BL/6 mice (6 months old, bodyweight 25-33 g, Nrf2 WT and KO) were obtained from the Model Animal Research Centre of Nanjing University (Nanjing, China). All mice were domesticated under a $12 \mathrm{~h}$ light/dark cycle at a controlled temperature $\left(25^{\circ} \mathrm{C}\right)$ with free access to food and tap water. Nrf2 WT mice were divided into 6 groups: the aging control, model control, AP 60 (60 mg/kg), and AP-SD groups (20, 40, and $60 \mathrm{mg} / \mathrm{kg})$. $\mathrm{Nrf} 2 \mathrm{KO}$ mice were divided into 3 groups: the aging control, model control, and AP-SD 60 groups $(60 \mathrm{mg} / \mathrm{kg})$. The doses were defined by the content of pure AP in the solid dispersion and were determined by preliminary experiments. Animals were treated according to the following procedures: aging control mice were fed normal diet for 9 months; model control mice were fed normal diet during months $1-3$, high-fat diet and intake of HQ dissolved in the drinking water $(0.8 \%)$ during months 4-6, normal diet and intragastric administration with $0.5 \%$ CMC-Na suspension daily during months 7-9. For the treated group mice, model control mice were intragastrically administrated with corresponding drugs (suspended in $0.5 \% \mathrm{CMC}-\mathrm{Na}$ ) at the last 3 months. All experimental procedures were approved by the institutional and local committee for the care and use of animals, and all animals received humane care according to the National Institutes of Health (USA) guidelines.

2.7. Fundus Autofluorescence. The mice were anesthetized using a ketamine $(120 \mathrm{mg} / \mathrm{kg}$ body weight) and xylazine $(8 \mathrm{mg} / \mathrm{kg}$ body weight) intraperitoneal injection, and the pupil was fully dilated with tropicamide phenylephrine eye drops (Kanda Pharmaceutical, Japan). The mouse was placed on the table with its head in the chinrest. To maintain corneal hydration and improve image quality, the viscoelastic material (Viscoat, Alcon-Couvreur, Belgium) was smeared on the cornea covering with a coverslip. A 90D noncontact slit lamp lens was fixated directly in front of the confocal scanning laser ophthalmoscope (Heidelberg Engineering, Heidelberg, Germany). Fluorescence was excited using a $488 \mathrm{~nm}$ argon laser or a $790 \mathrm{~nm}$ diode laser. As Charbel Issa did [21], images were recorded using the ART mode for the quantitative analysis of fundus autofluorescence (FAF); the mean grey level on mouse FAF images was measured using ImageJ software (version 1.52, NIH, Bethesda, USA).

2.8. Optical Coherence Tomography. Like the FAF assessment, the animal was anesthetized, the pupil fully dilated, and the viscoelastic material was smeared on the cornea covering with a coverslip; then, a 90D noncontact slit lamp lens was placed in front of an optical coherence tomography (OCT) device (Cirrus HD-OCT 4000, Carl Zeiss Meditec, Dublin, CA); cross-sectional images of the retina were undertaken. The average thickness of the mouse retina was obtained using the macular cube scan mode according to the methods reported by Song [22].

2.9. Transmission Electron Microscopy. Eyeballs were fixed in $4 \%$ paraformaldehyde for $20 \mathrm{~min}$. The cornea, lens, and vitreous were removed. The yye wall tissue $(2 \times 4 \mathrm{~mm})$ was excised from the bilateral area of the optic disc and fixed with glutaral/osmic acid, coated with epoxy resins, and sectioned. After double staining with uranyl acetate and lead citrate, the sections were examined with transmission electron microscope (Tecnai G2 Spirit BioTWIN; FEI, Hillsboro, OR, USA) and images were taken. The area of the sediment beneath RPE was determined according to the methods reported by Edward [23]. Briefly, images were opened in ImageJ software and calibrated using the embedded scale bar. The region of the sediment was drawn using free selection tool, and the area was measured. The thickness of the Bruch membrane (BrM) was directly measured by electron microscopy.

2.10. Measurements of ROS, MDA, and Antioxidant Enzymes. The retina was isolated, and tissue homogenates were prepared via centrifugation at $4^{\circ} \mathrm{C}, 3000 \mathrm{r} / \mathrm{min}$ for 10 min. Levels of ROS were determined using the DCFHDA method. Levels of MDA were measured using the thiobarbituric acid method. In addition, activities of SOD and GSH-PX were measured using the corresponding enzymelinked immunosorbent assay kits at the wavelength of 450 and $412 \mathrm{~nm}$, respectively. Kits above were from Nanjing Jiancheng Bioengineering Institute (Nanjing, China), and experiments were performed according to the instructions provided by the manufactures.

2.11. Western Blot Analysis. The mouse retina/choroid tissue was lysed in the RIPA buffer containing a protease inhibitor.

For examining the Nrf2 expression, nuclear and cytoplasmic proteins were separated using a Bioepitope Nuclear and Cytoplasmic Extraction Kit (Bioworld Technology, St. Louis Park, MN, USA) following the manufacturer's guidelines. Proteins $(50 \mu \mathrm{g} /$ well) were separated by SDS-polyacrylamide gel and transferred to a PVDF membrane (Millipore, Burlington, 
MA, USA). After blocking with 5\% skim milk in Tris-buffered saline containing $0.1 \%$ Tween 20 , the membranes were incubated with primary corresponding primary antibodies. The blots were then incubated with a secondary antibody (antirabbit horseradish peroxidase-conjugate/anti-mouse HRPconjugate), and the protein bands were visualized using a chemiluminescence reagent (Millipore, Burlington, MA, USA). Equivalent loading was confirmed using an antibody against GAPDH for total proteins and against Lamin B for nuclear proteins. The levels of target protein bands were densitometrically determined using Quantity One 4.4.1.

2.12. Statistical Analysis. Data were presented as mean $\pm \mathrm{SD}$, and results were analyzed using GraphPad Prism 5 software. The significance of difference was determined by Student's $t$-test for comparison between two groups and one-way ANOVA with post hoc Tukey's test for comparison between multiple groups. A value of $p<0.05$ was considered to be statistically significant.

\section{Results}

3.1. AP-SD Increases the Solubility and Dissolution of AP In Vitro. We determined the equilibrium solubility of AP and AP-SD in water and chloroform. The results showed that the equilibrium solubility of AP-SD in both water and chloroform was significantly higher than that of AP (Table 1). Determination of the dissolution rate showed that AP-SD had significantly higher cumulative dissolution rates than $\mathrm{AP}$ at each time points (Figure 1).

3.2. AP-SD Enhances the Absorption of AP In Vivo. We obtained the plasma concentration-time curve of AP and AP-SD in rats (Figure 2). $C_{\max }, T_{\max }$, and $\mathrm{AUC}_{0 \sim 24}$ for $\mathrm{AP}$ and AP-SD were calculated with ImageJ software (Table 2). The results showed that AP-SD had better bioavailability than AP.

3.3. AP-SD Alleviated Pathological Changes of the Retina. In clinical, fundus AF and OCT are the most used noninvasive means for monitoring of dry AMD [24, 25]. Fundus $\mathrm{AF}$ generated with wavelength between 500 and $750 \mathrm{~nm}$ is dominated by RPE lipofuscin, a complex mixture of fluorophores being accumulated in the RPE after phagocytosis of POS [26]. Therefore, AF intensity indicates the level of lipofuscin in vivo in the RPE. Spectral-domain- (SD-) OCT provides high-quality, cross-sectional images of the retina including RPE with resolution approaching histology performed with light microscopy [27]. To evaluate the therapeutic effects of AP-SD, we established a dry AMD mouse model that mimics three risk factors for AMD in humans: aging, hyperlipidemia, and smoking (HQ is abundant in cigarette smoke) [28]. HQ, an electrophilic, could inhibit the binding of BACH1 with Nrf2, thus activating the Nrf2 pathway [29]. Our results showed that AF intensity in model mice was significantly enhanced compared with that in aging mice, and in Nrf2KO mouse higher than in Nrf2WT mice. AF intensity was attenuated after treatment with AP-SD in Nrf2WT mice (Figures 3(a) and 3(b)). Correspondingly, the images of OCT scanning showed that the outer layer struc-
TABLE 1: Equilibrium solubility of AP of both dosage forms $\left(37^{\circ} \mathrm{C}\right.$, $n=3)$.

\begin{tabular}{lcc}
\hline \multirow{2}{*}{ Solvents } & \multicolumn{2}{c}{ Concentration $(\mu \mathrm{g} / \mathrm{mL})$} \\
& AP & AP-SD \\
\hline Water & $1.33 \pm 0.15$ & $352.09 \pm 22.56^{* *}$ \\
Chloroform & $2.18 \pm 0.21$ & $1.43 \times 10^{3} \pm 187.11^{* *}$ \\
\hline
\end{tabular}

Significance: ${ }^{* *} p<0.01$ versus AP.

ture of the retina including photoreceptors, RPE, and Bruch membrane $(\mathrm{BrM})$ became unclear, and the retina was thinner in model mice compared with aging mice, more significant in Nrf2 KO mice than in Nrf2 WT mice (Figure 4). Treatment with AP-SD could restore the retinal structure in Nrf2WT mice.

We further observed the retinal ultrastructure; the results demonstrated that there were undigested phagosomes (Figure 5(a)) and autophagosome (Figure 5(b)) present in the RPE of the model mouse. There were obvious sediments under RPE and thickened BrM in the model mice compared to the aging mice, and it is more severe in Nrf2 $\mathrm{KO}$ mice. AP$\mathrm{SD}$ reduced the area of the sediment and thinned BrM in Nrf2WT mice (Figure 6). In particular, AP-SD only alleviated retinopathy in Nrf2 WT mice and had no effects in Nrf2 KO mice. In addition, the therapeutic effect of AP-SD was dose dependent, and AP did not work. Collectively, these data indicated that AP-SD more potently improved pathological changes of the retina in Nrf2 WT model mice with dry AMD.

3.4. AP-SD Induced Nrf2 Nuclear Translocation and Increased Antioxidant Gene Expression. To explore the underlying mechanism for AP-SD reducing retinopathy in model mice with dry AMD, we first assessed the effects of AP-SD on Nrf2 pathway. As shown in Figure 7(a), the level of nuclear Nrf2 was increased and the level of cytoplasmic Nrf2 was reduced in model mice compared with those in aging mice. AP-SD decreased the level of cytoplasmic Nrf2 and increased the level of nuclear Nrf2 in a dose-effect manner, indicating that AP-SD promoted Nrf2 nuclear translocation (Figure 7(b)). Furthermore, we examined the ability of AP-SD to upregulate the expressions of the targeted genes of Nrf2, upon its increased nuclear translocation. The expressions of HO-1 and NQO-1 were upregulated in the Nrf2 WT model mice and downregulated in the Nrf2 KO model mice, compared with those in aging mice (Figure 8(a)). AP-SD dose dependently increased the expressions of HO-1 and NQO-1 in Nrf2 WT mice but had no effects in Nrf2 KO mice as expected (Figure 8(b)).

3.5. AP-SD Restored Activities of SOD and GSH-Px Enzyme and Decreased Levels of SOD and MDA. We measured the activity of SOD and GSH-Px enzyme in mouse, two important antioxidant enzymes of the retina. The results showed that the activities of SOD and GSH-Px were significantly decreased in Nrf2 WT model mice and are more remarkable in Nrf2 KO model mice. AP-SD dose dependently restored 




FIGURE 1: AP-SD had significantly higher cumulative dissolution rates than AP. Determination of cumulative dissolution rates of AP and AP$\mathrm{SD}$ at indicated time points. Data are means \pm standard deviation $(n=6)$.

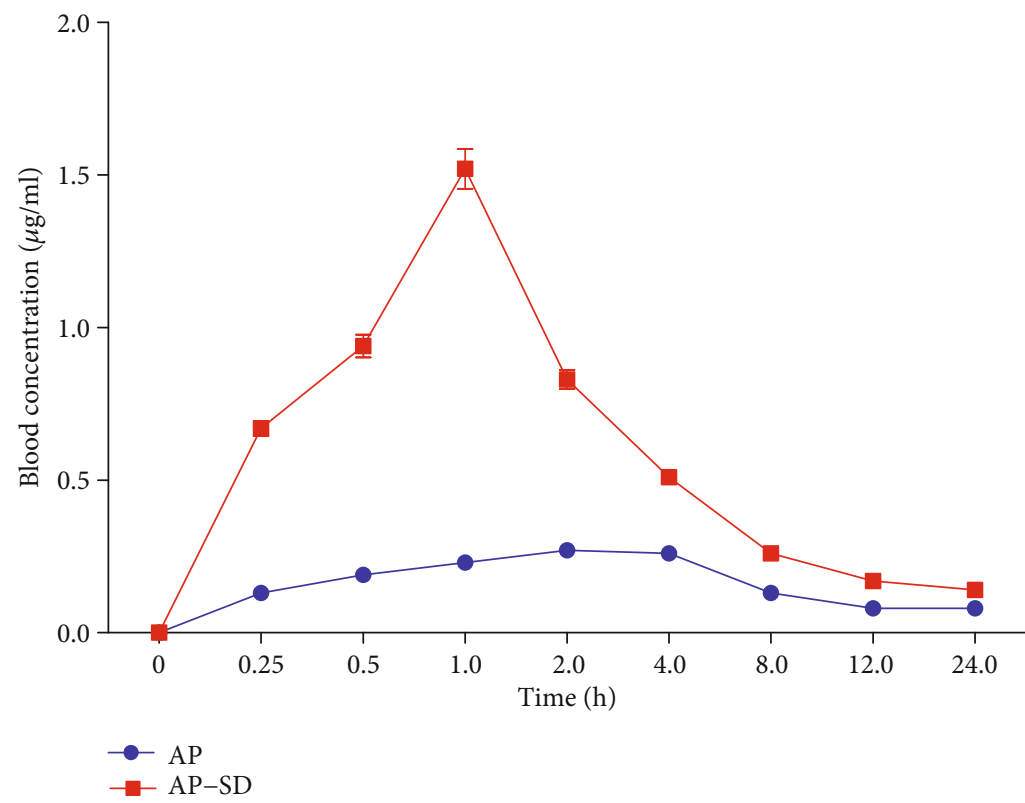

FIGURE 2: AP-SD had better oral bioavailability than AP. Determination of blood concentrations of AP and AP-SD at indicated time points in rats. Data are means \pm standard deviation $(n=6)$.

TABLE 2: Pharmacokinetic parameters of AP after oral administration of pure AP and AP-SD in rats (dose $50 \mathrm{mg} / \mathrm{kg}, n=$ 6 , mean \pm SD).

\begin{tabular}{lcc}
\hline Parameters & AP & AP-SD \\
\hline$C_{\max }(\mu \mathrm{g} / \mathrm{mL})$ & 0.27 & $1.52^{* *}$ \\
$T_{\max }(\mathrm{hr})$ & 2.0 & $1^{*}$ \\
$\mathrm{AUC}_{0-24}(\mu \mathrm{gh} / \mathrm{mL})$ & 3.10 & $7.68^{* *}$ \\
\hline
\end{tabular}

Significance: ${ }^{*} p<0.05,{ }^{* *} p<0.01$ versus AP. the activities of SOD and GSH-Px in Nrf2 WT mice, not in Nrf2 KO mice (Figures 9(a) and, 9(b)). ROS is a by-product of cellular oxidative phosphorylation and its level is consistent with oxidative stress. ROS causes lipid peroxidation of the biomembrane, which leads to the production of a large amount of MDA [2]. Therefore, we examined the levels of ROS and MDA in the mouse retina. The results showed that the levels of ROS and MDA were significantly elevated in Nrf2 WT and Nrf2 KO model mice, but higher in Nrf2 KO mice. AP-SD decreased the ROS and MDA levels in dose- 




Aging

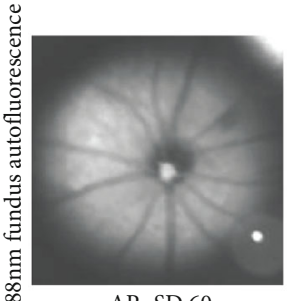

AP-SD 60



Aging

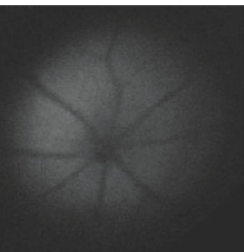

Aging

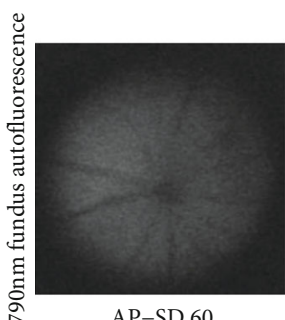

AP-SD 60

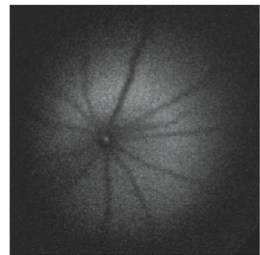

Aging

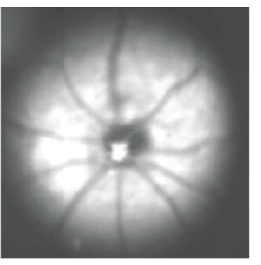

Model

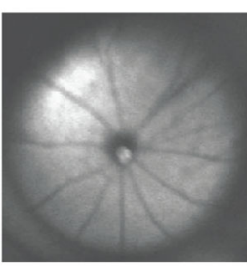

AP-SD 40

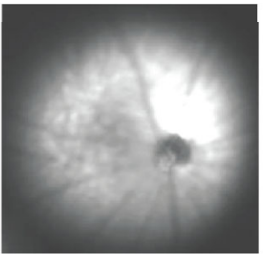

Model

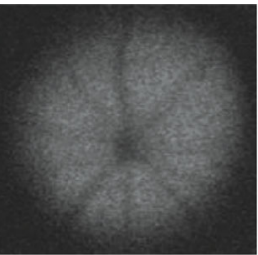

Model

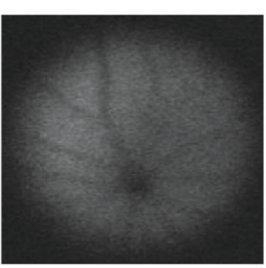

AP-SD 40

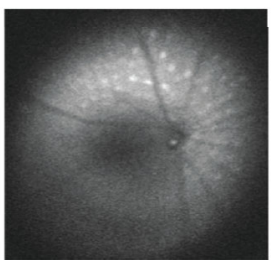

Model

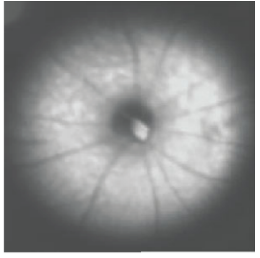

AP 60

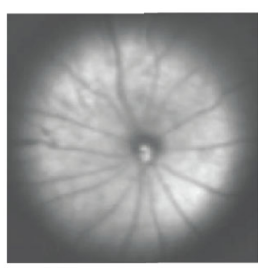

AP-SD 20

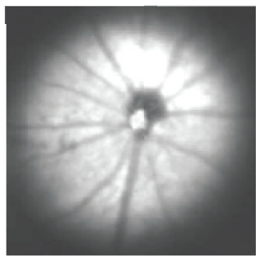

AP-SD 60

(a)

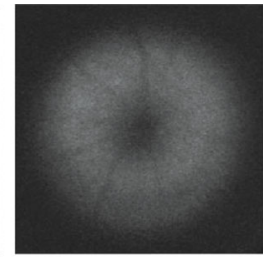

AP 60

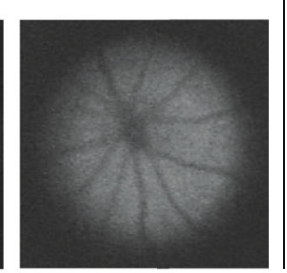

AP-SD 20

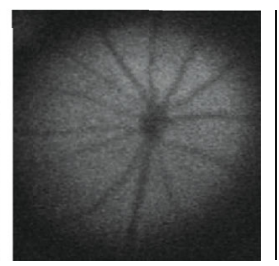

AP-SD 60

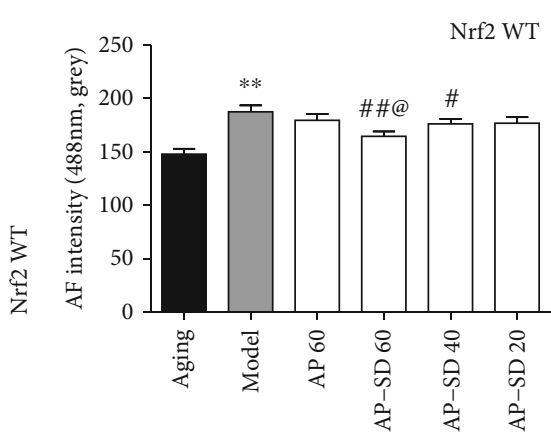

Nrf2 KO
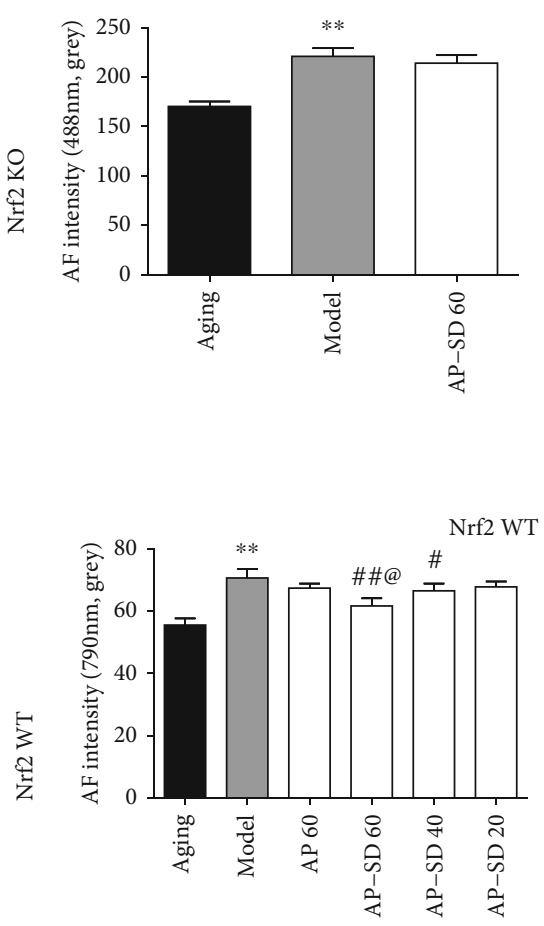

Nrf2 KO

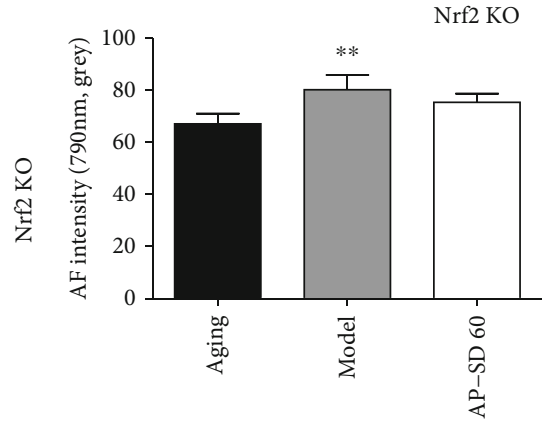

(b)

FIgURE 3: Representative images of fundus AF excited at 488 (a) and $790 \mathrm{~nm}$ (b) in Nrf2 WT and KO mice. AF intensity in model mice was significantly enhanced compared with that in aging mice, and in Nrf2 KO mice higher than in Nrf2 WT mice. AF intensity was decreased after treatment with AP-SD in Nrf2 WT mice, but not in Nrf2 KO mice. ${ }^{* *} p<0.01$, model control versus aging control; ${ }^{\#} p<0.05$, ${ }^{\# \#} p<0.01, \mathrm{AP}-$ SD versus model control; ${ }^{\circledR} p<0.05$, AP-SD 60 versus AP-SD 40. Data are means \pm standard deviation $(n=5)$. 


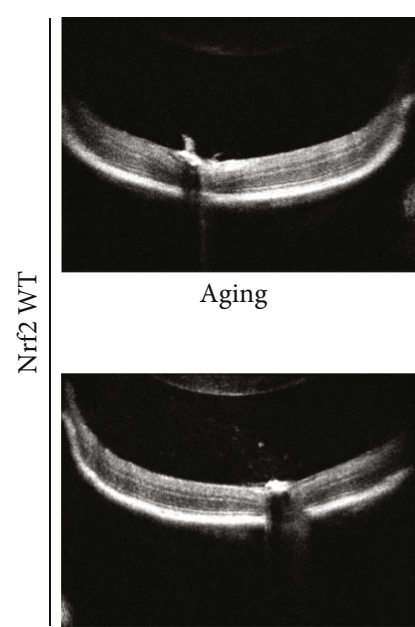

AP-SD 60

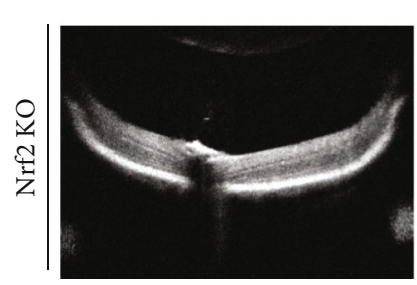

Aging

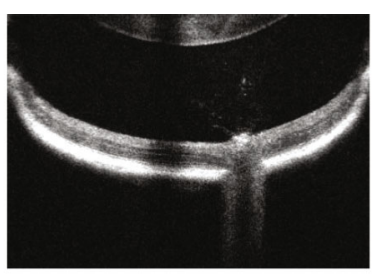

Model



AP-SD 40



Model



AP 60



AP-SD 20

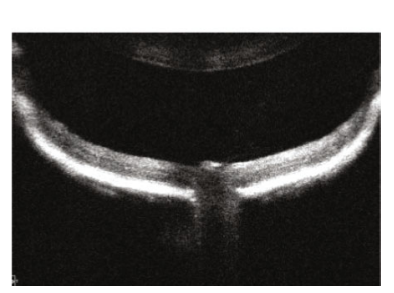

AP-SD 60



$\mathrm{Nrf2} \mathrm{KO}$

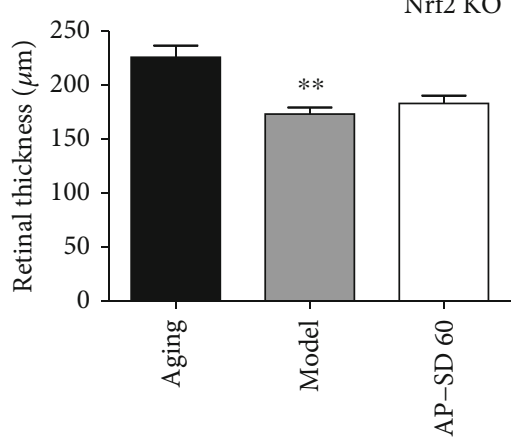

FIGURE 4: Representative images of OCT in Nrf2 WT and KO mice. Outer layer structure of the retina was unclear and the retina was thinner in model mice compared with aging mice, more significant in Nrf2 KO mice. Treatment with AP-SD dose dependently restored the retinal structure in Nrf2 WT mice and did not in Nrf2 KO mice. ${ }^{* *} p<0.01$, model control versus aging control; ${ }^{\#} p<0.05,{ }^{\# \#} p<0.01$, AP-SD versus model control; ${ }^{@ @ ~} p<0.01$, AP-SD 60 versus AP-SD 40. Data are means \pm standard deviation $(n=5)$.
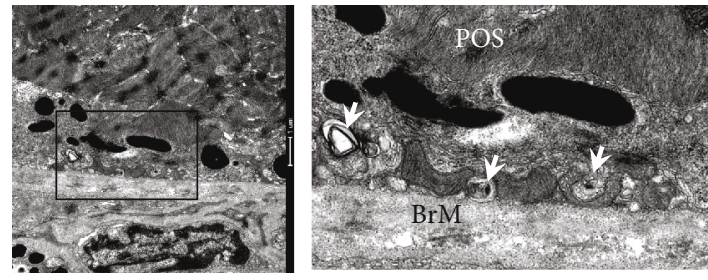

(a)
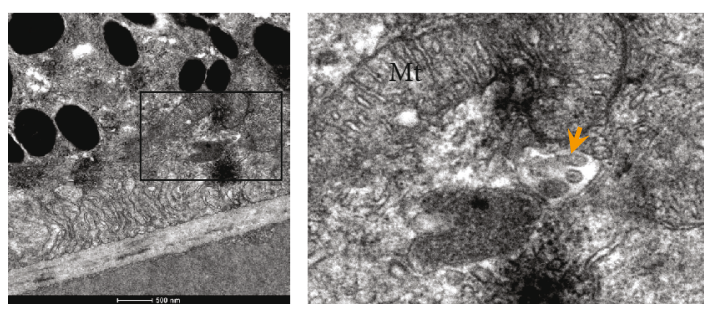

(b)

FIGURE 5: Transmission electron microscopy showed that there were undigested phagosomes (a, white arrow) and autophagosome (b, orange arrow) present in the RPE of the model mouse. POS: photoreceptor outer segment; Mt: mitochondria; BrM: Bruch membrane. effect manner in Nrf2 WT mice, not in Nrf2 KO mice (Figures 10(a) and 10(b)).

3.6. AP-SD Increased p62 Protein Expression and Regulated Autophagy in an Nrf2-Dependent Manner. The p62 protein and LC3 have been widely regarded as markers for autophagic activity. p62 is used as a marker of autophagic degradation [30] and LC3-II serves as a marker for autophagosome formation [31]. Autophagy increases often coincides with the induction of the Nrf2 pathway in stressed conditions [32]. Based on these findings, we assessed whether Nrf2 directly upregulated the expression of p62. As shown in Figure 10(a), the upregulation of p62 expression was only found in Nrf2 WT model mice, but not in Nrf2 KO model mice, compared with the aging mice. Under the same conditions, Nrf2 levels in the nucleus significantly increased in Nrf2 WT model mice, as shown in the previous results. Our findings are consistent with those of several reports $[33,34]$. In addition, AP-SD dose dependently increased p62 expression in Nrf2 WT mice, not in $\mathrm{Nrf} 2 \mathrm{KO}$ mice (Figure 11(b)). Meanwhile, the upregulation of LC3 II expression was also only shown in Nrf2 WT mice, but not in Nrf2 $\mathrm{KO}$ mice, compared with the aging mice. In Nrf2 WT mice (Figure 11(a)), the expression of LC3 II increased in dose-dependent manner after treatment with AP-SD, 


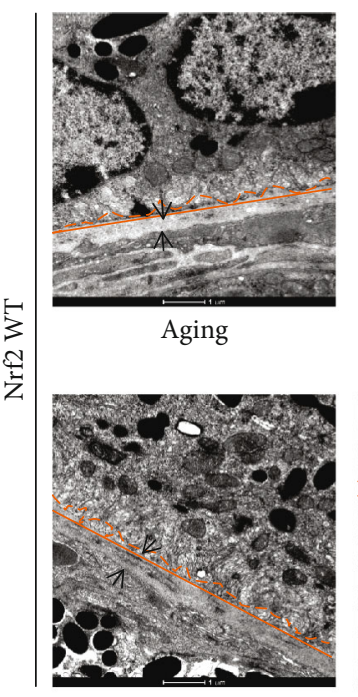

AP-SD 60

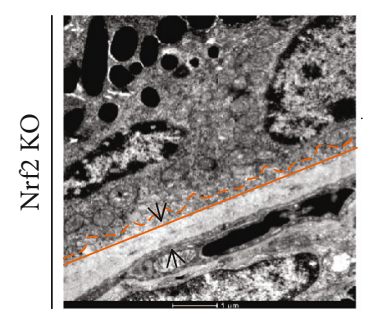

Aging

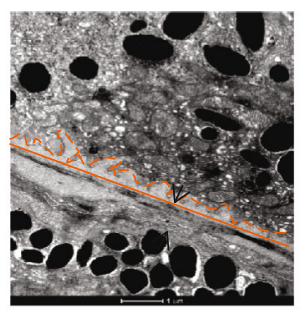

Model

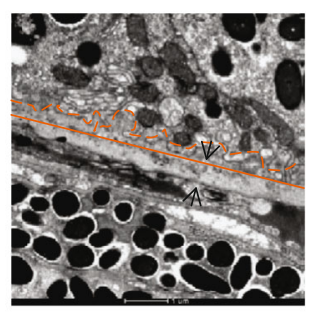

AP-SD 40

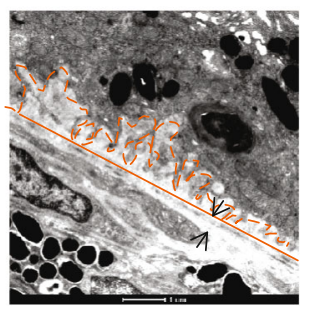

Model
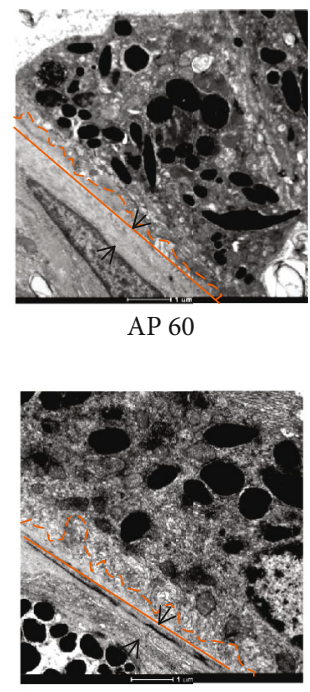

AP-SD 20



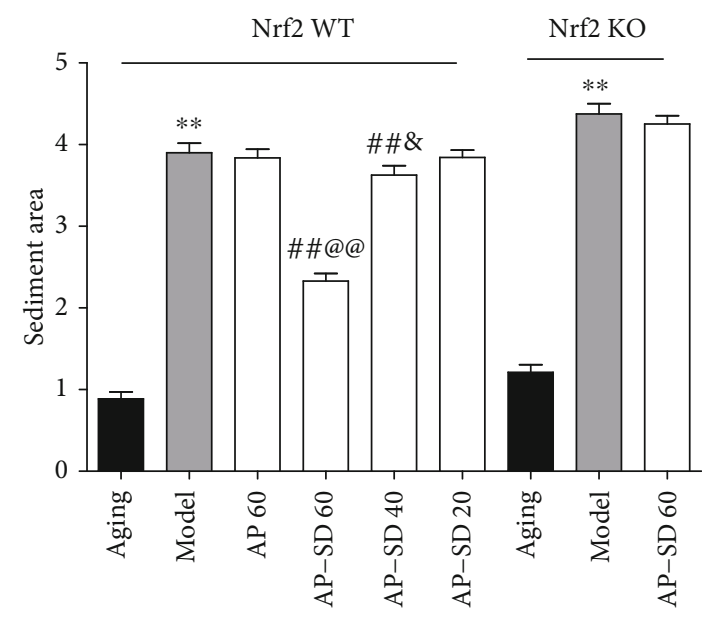



FIGURE 6: Representative images of TEM in Nrf2 WT and KO mice. There were obvious sediments under RPE (orange area) and thickened $\mathrm{BrM}$ (black arrow) in model mice compared to the aging mice, and it is more severe in Nrf2 KO mice. AP-SD reduced the area of the sediment and thinned BrM in Nrf2 WT mice and not in Nrf2 KO mice. ${ }^{* *} p<0.01$, model control versus aging control; ${ }^{\# \#} p<0.01$, AP-SD versus model control; ${ }^{@} p<0.05,{ }^{@} @ p<0.01$, AP-SD 60 versus AP-SD $40 ;{ }^{\circledR} p<0.05$, AP-SD 40 versus AP-SD 20 . Data are means \pm standard deviation $(n=5)$.

but there was no significant change in Nrf2 KO mice (Figure 11(b)). Together, these results imply that Nrf2 is a mediator of the activation of autophagy in the model mice with dry AMD.

\section{Discussion}

Up to now, no therapy options are approved for the treatment of dry AMD. Several pathways, including oxidative stress, deposits of lipofuscin, and chronic inflammation, seem to play important roles in the pathogenesis of dry AMD and represent possible targets [35]. Oxidative stress has been suggested to be a critical component of AMD pathogenesis. Many reports demonstrated that natural products are able to decrease the occurrence of several diseases induced by oxidative injury, including AMD [36, 37]. Dietary supplements containing high dose of antioxidants and minerals (vitamins $\mathrm{C}$ and $\mathrm{E}, \beta$-carotene, and zinc) delayed the progression of AMD intermediate to advanced stages [38]. Quercetin, lutein, zeaxanthin, and hesperetin have also been shown to have the potential to prevent AMD progress [39-
41]. It has been reported that AP plays a protective role in treating diseases associated with the oxidative process, such as cardiovascular and neurological disorders [42, 43]. However, like other flavonoids, AP has poor solubility in water and fat, therefore having low bioavailability. PVP, a hydrophilic excipient, could convert drugs to an amorphous form, and immensity enhances the solubility and dissolution of drugs $[44,45]$. In this study, we prepared AP-SD with PVP $\mathrm{K} 30$; the results showed that the formation of solid dispersion increased the solubility and dispersion of AP, so the oral bioavailability of AP-SD was better than that of AP in rats. We then used dry AMD model mouse to verify the benefits of AP-SD in improving bioavailability. The results showed that AP-SD could reduce retinopathy, while AP could not, indicating that the formation of solid dispersion reasonably enhanced the protective effects of AP on the mouse retina on account of the increased bioavailability.

Recently, the Nrf2 pathway was considered a key contributor to the response to increased oxidative stress in RPE. Nrf2 knockout mouse has been shown to induce AMD-like pathological changes, manifested by the accumulation of 

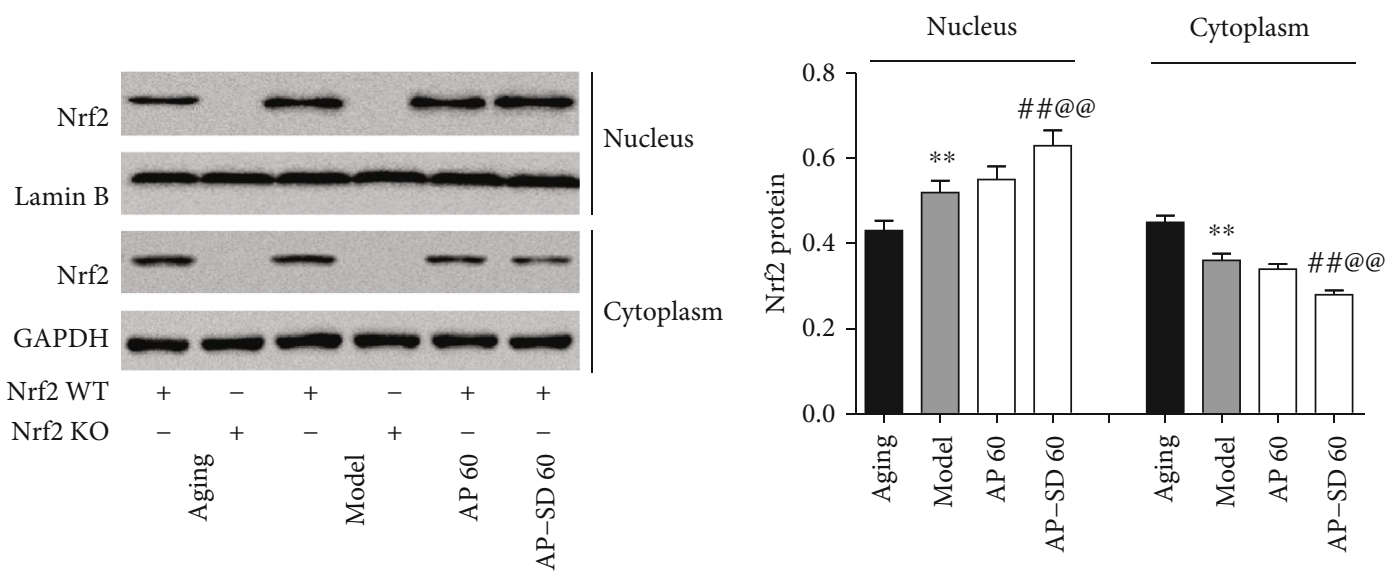

(a)
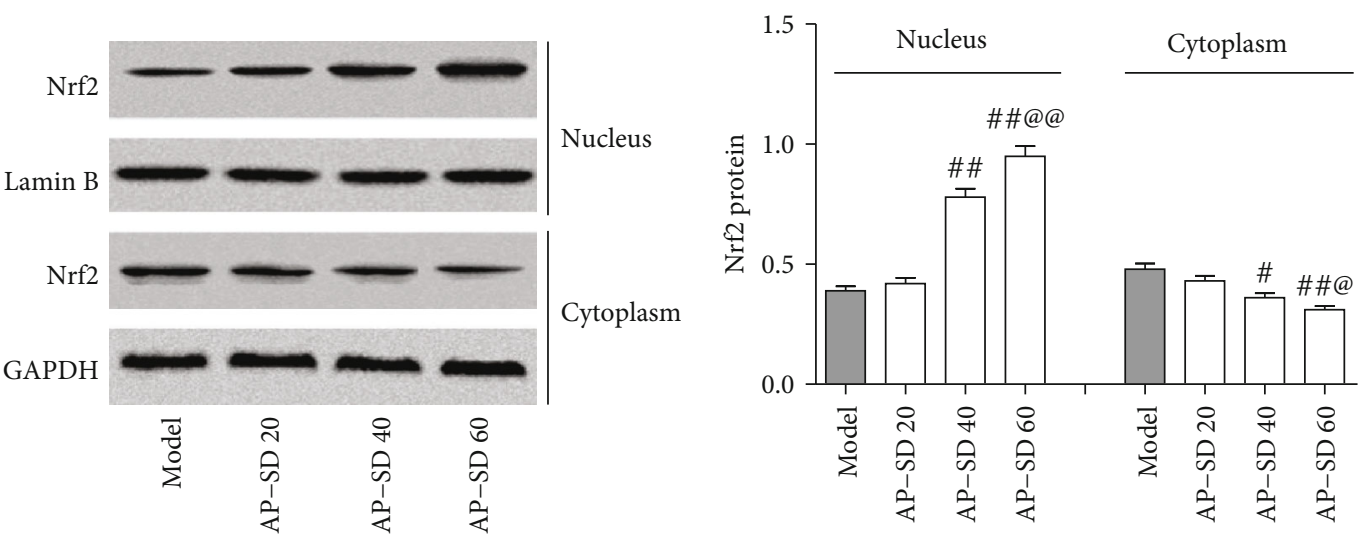

(b)

FIgURE 7: AP-SD promoted Nrf2 nuclear translocation. (a) The level of nuclear Nrf2 was increased and the level of cytoplasmic Nrf2 was reduced in model mice compared with those in aging mice. (b) AP-SD decreased the level of cytoplasmic Nrf2 and increased the level of nuclear Nrf2. ${ }^{* *} p<0.01$, model control versus aging control; ${ }^{\# \#} p<0.01$, AP-SD versus model control; ${ }^{@} p<0.05,{ }^{@} \mathrm{p}<0.01, \mathrm{AP}-\mathrm{SD} 60$ versus AP-SD 40. Data are means \pm standard deviation $(n=5)$.

lipofuscin, drusen, choroidal neovascularization and accumulation of the autophagosome [46]. In this study, similar lesions were showed in the retina of the model mouse. Activation of the Nrf2 pathway could have a therapeutic potential in protecting RPE cells against oxidative stress, so it may be beneficial for dry AMD [47]. Based on this point, we have previously reported that AP protected ARPE-19 cells against oxidative injury by the activation of Nrf2 signaling in vitro. In the present study, the effects of AP were validated in model mouse of dry AMD in vivo. The upregulation of Nrf2 expression and nuclear translocation were observed in Nrf2 WT mice. AP-SD promoted Nrf2 nuclear translocation, upregulated the expression of $\mathrm{Nrf} 2$ and targeted protein HO-1 and GCL, restored activities of SOD and GSH-PX, and decreased the levels of ROS and MDA in Nrf2 WT mice. As expected, similar results were not observed in Nrf2 KO mice. In conclusion, our findings suggested that AP-SD protected the retina against oxidative injury via activation of the Nrf2 signaling pathway.

It was reported that AP regulate autophagy. For example, AP promoted autophagy through the mTOR/AMPK/ULK1 pathway and could play an antidepressant effect [48], inhibited the growth of cisplatin-resistant colon cancer cells by inducing autophagy and apoptosis [49], and restored impairment of autophagy and downregulation of unfolded protein response regulatory proteins in keratinocytes exposed to ultraviolet B radiation [50]. Studies have suggested that p62 protein is regulated by oxidative stress and is a transcriptional target of Nrf2 $[51,52]$. This study focused on the effect of AP on autophagy pathway in the retina of model mice and the relationship between the p62 expression, autophagy, and Nrf2 pathway. The results showed that the expression of p62 and LC3 in Nrf2 KO mice were significantly lower than that in Nrf2 WT mice. In Nrf2 WT mice, AP-SD dose dependently upregulated the expression of p62 and LC3 but had no effect in Nrf2KO mice. Our finding suggested that APSD upregulated the autophagy in an Nrf2-dependent manner. It has been well known that the nuclear translocation of Nrf2 is increased and the expression of Nrf2 is upregulated in oxidative stress. Nrf2 could regulate the transcription of autophagy genes, such as p62, ULK1, Atg7 and GABARAPL1, Atg2B, Atg5, and Atg4D, because there is an ARE sequence in their promoter regions. The expressions of several autophagy markers (including LC3) were decreased in Nrf2-deficient mice $[33,53]$. However, it has been reported that trehalose upregulate Nrf2 and autophagy in a p62- 



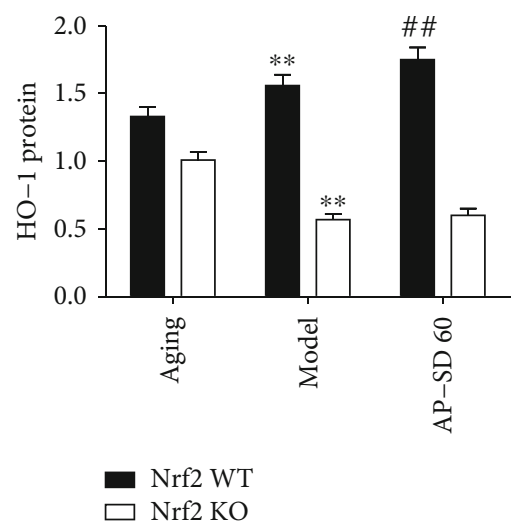

(a)

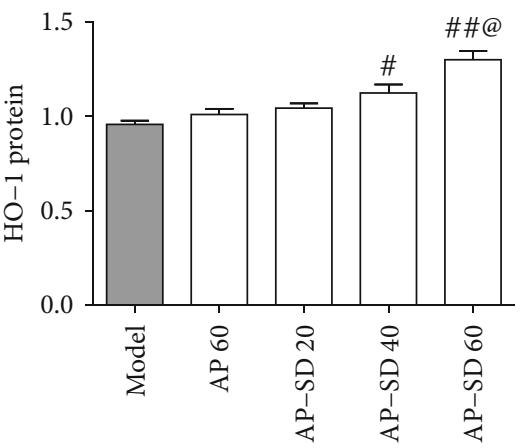

(b)
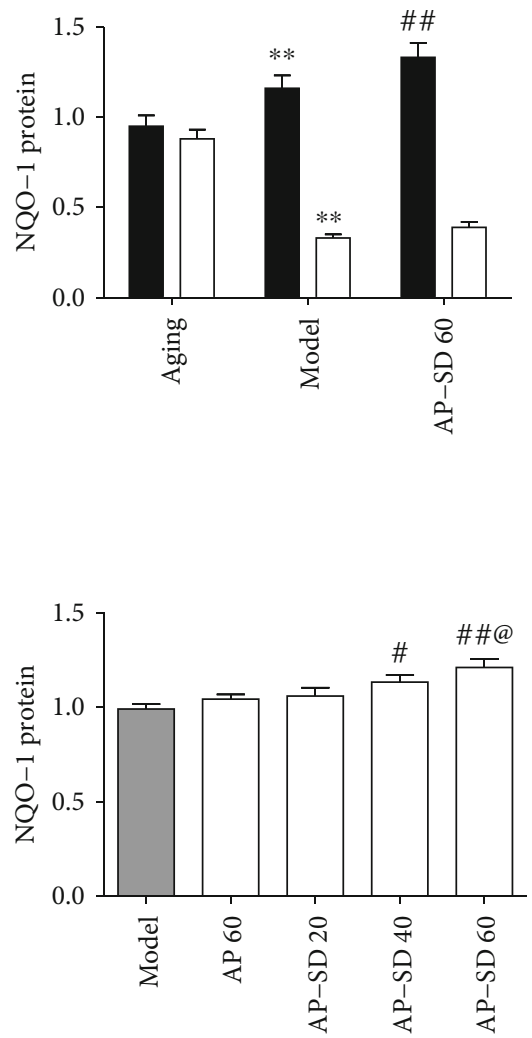

FIgURE 8: AP-SD upregulated the expressions of the targeted genes of Nrf2. (a) The expressions of HO-1 and NQO-1 were upregulated in the Nrf2 WT model mice and downregulated in Nrf2 KO model mice, compared with those in aging mice. (b) AP-SD increased the expressions of HO-1 and NQO-1 in Nrf2 WT mice but not in Nrf2 KO mice. ${ }^{* *} p<0.01$, model control versus aging control; ${ }^{\#} p<0.05,{ }^{\# \#} p<0.01$, AP-SD versus model control; ${ }^{@} p<0.05$, AP-SD 60 versus AP-SD 40. Data are means \pm standard deviation $(n=5)$.

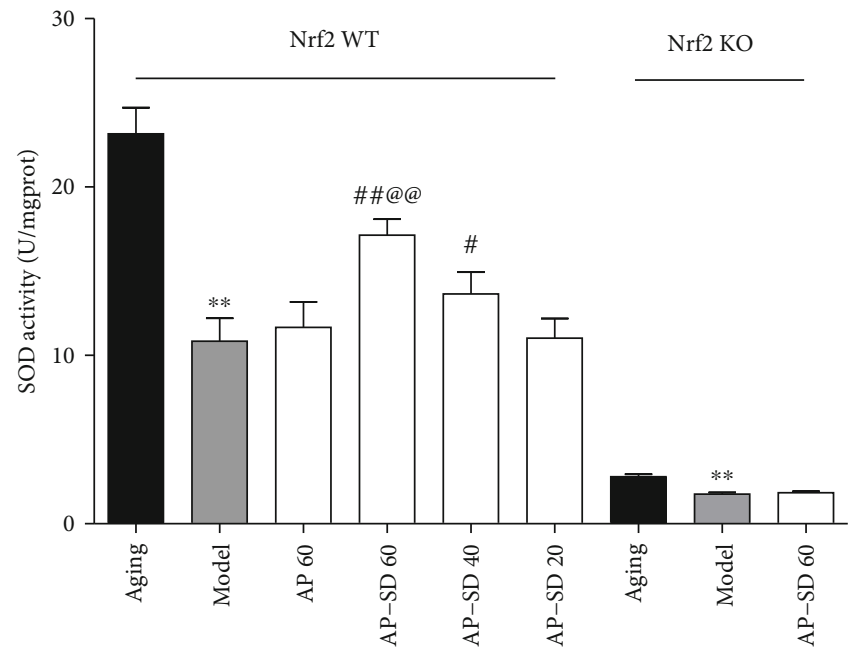

(a)

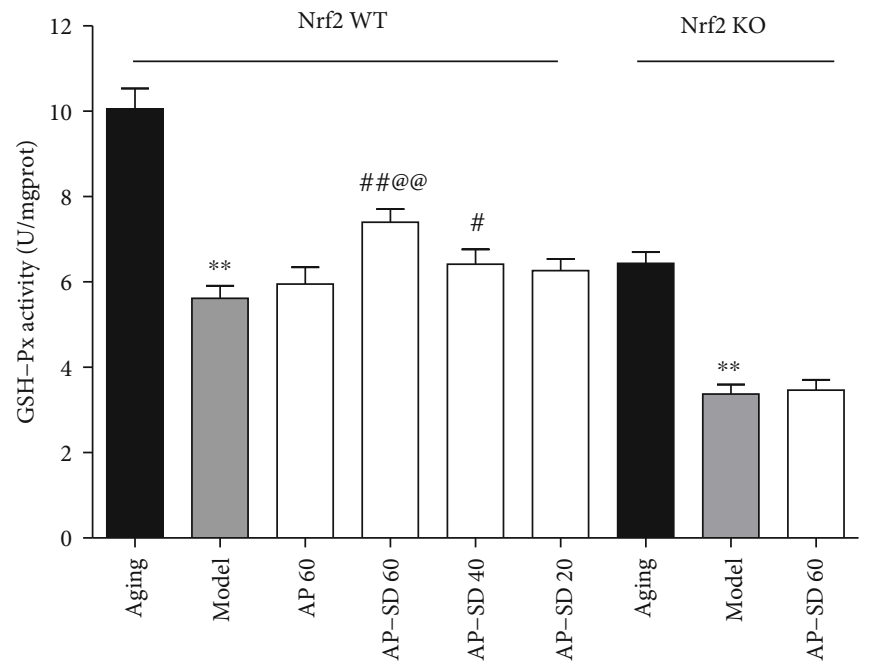

(b)

FIGURE 9: AP-SD restored the activities of SOD and GSH-Px. The activities of SOD (a) and GSH-Px (b) were decreased in Nrf2 WT model mice and more remarkable in Nrf2 KO model mice. AP-SD restored the activities of SOD and GSH-Px in Nrf2 WT mice, not in Nrf2 KO mice. ${ }^{* *} p<0.01$, model control versus aging control; ${ }^{\#} p<0.05$, ${ }^{\# \#} p<0.01$, AP-SD versus model control; ${ }^{@} p<0.01$, AP-SD 60 versus APSD 40. Data are means \pm standard deviation $(n=5)$. 




(a)

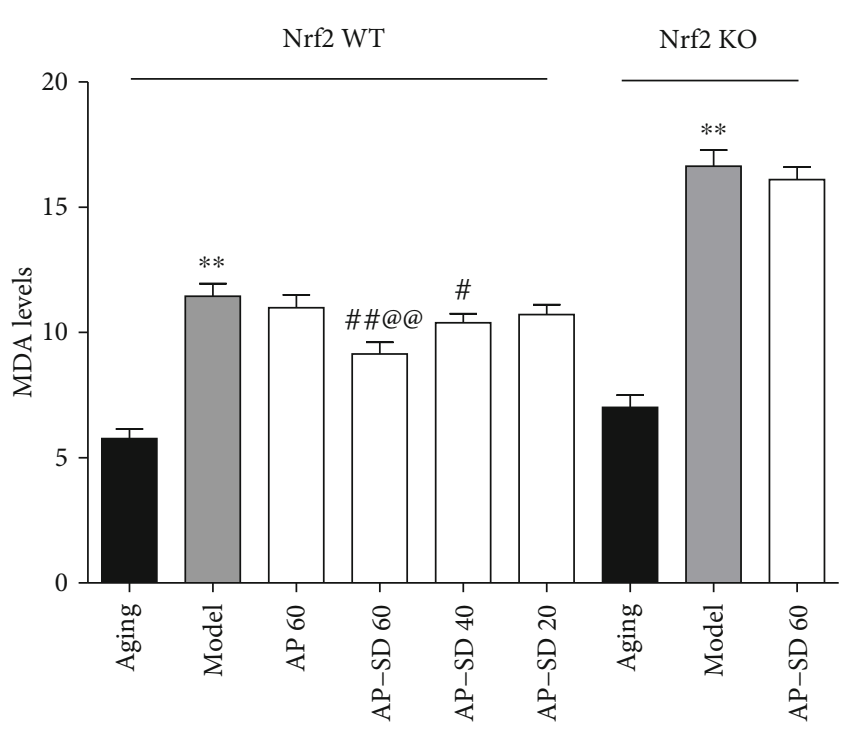

(b)

FIGURE 10: AP-SD decreased the ROS and MDA levels. The levels of ROS (a) and MDA (b) were significantly elevated in Nrf2 WT and Nrf2 KO model mice, but higher in Nrf2 KO mice. AP-SD decreased the ROS and MDA levels in Nrf2 WT mice, not in Nrf2 KO mice. ${ }^{* *} p<0.01$, model control versus aging control; ${ }^{\#} p<0.05,{ }^{\# \#} p<0.01$, AP-SD versus model control; ${ }^{@} p<0.01$, AP-SD 60 versus AP-SD 40. Data are means \pm standard deviation $(n=5)$.
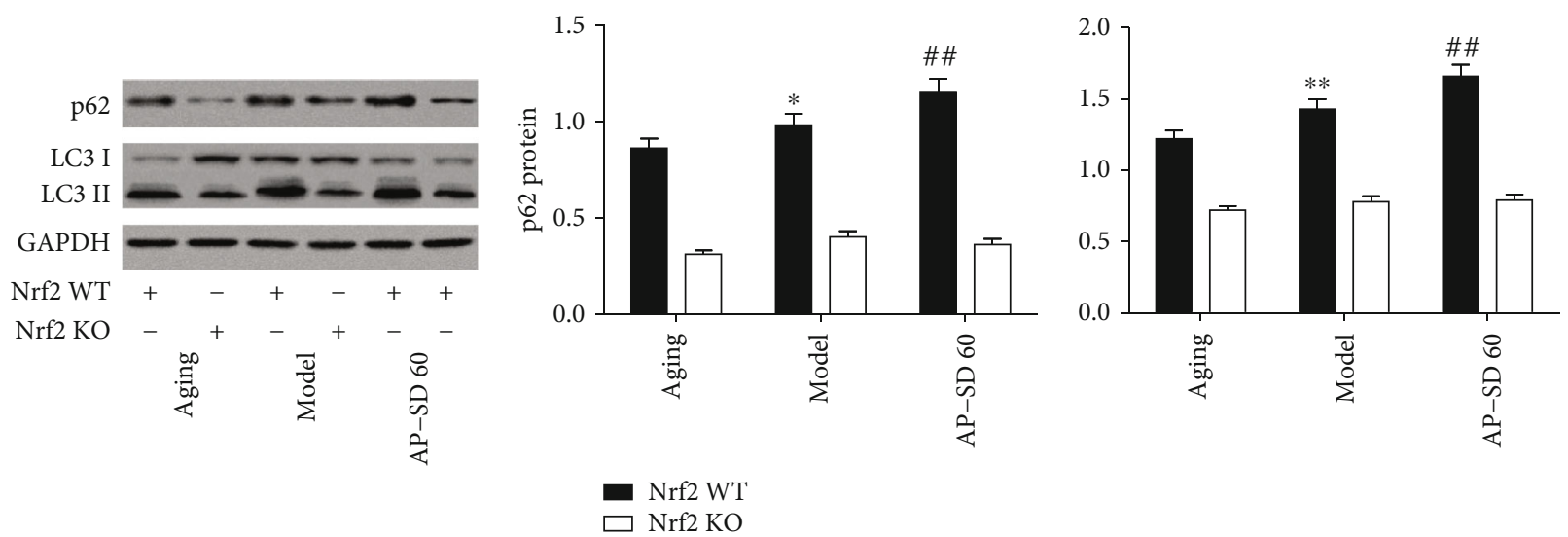

(a)
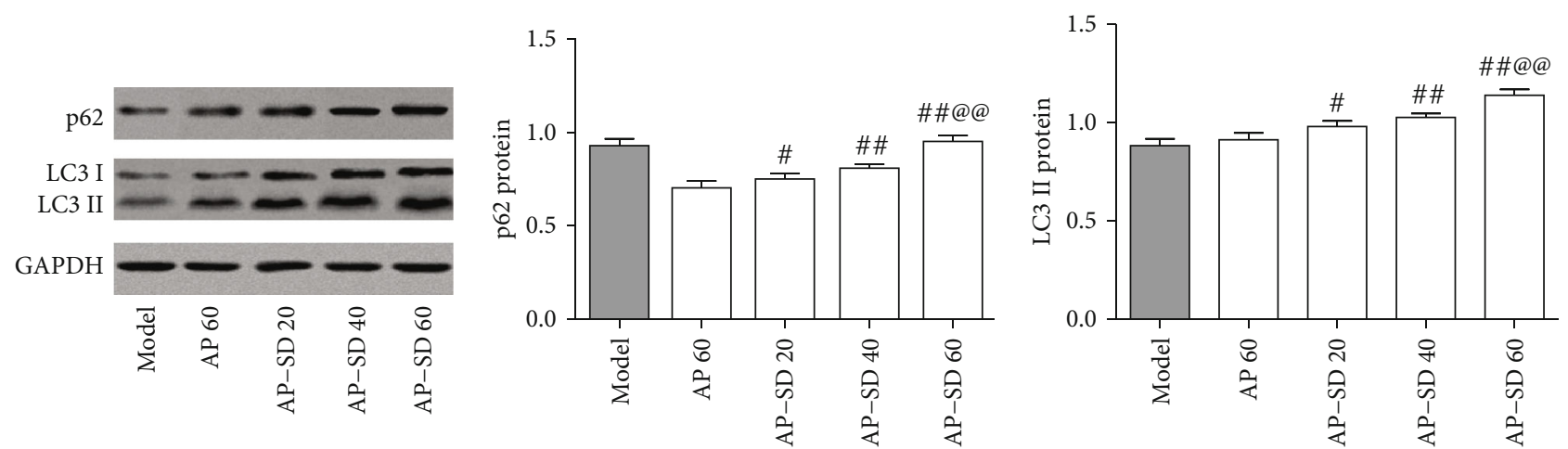

(b)

FIGURE 11: AP-SD regulated autophagy. (a) The expressions of p62 and LC3 II were upregulated in Nrf2 WT model mice, but not in Nrf2 KO model mice, compared with those in aging mice. (b) AP-SD increased the expressions of p62 and LC3 II in Nrf2 WT mice, but not in Nrf2 KO mice. ${ }^{*} p<0.05,{ }^{* *} p<0.01$, model control versus aging control; ${ }^{\#} p<0.05,{ }^{\# \#} p<0.01$, AP-SD versus model control; ${ }^{@ @ ~} p<0.01$, AP-SD 60 versus AP-SD 40. Data are means \pm standard deviation $(n=5)$. 
dependent manner in oxidative stress [12]. Therefore, the underlying mechanism of AP regulating the Nrf2 pathway and autophagy remains to be further studied.

In summary, AP-SD significantly enhanced the bioavailability of the original drug and reduced retinal oxidative injury in model mouse of dry AMD in vivo. Meanwhile, we provided evidence that AP not only activates Nrf2 pathway but also upregulates p62 and autophagy. AP-SD upregulated the expressions of antioxidant enzymes through the Nrf2 pathway and upregulated the autophagy in an Nrf2dependent manner to suppress retinal oxidative damage. The results suggest that AP-SD is a potential compound for the treatment of dry AMD.

\section{Data Availability}

The data used to support the findings of this study are included within the article.

\section{Disclosure}

The authors declare that the research was conducted in the absence of any commercial or financial relationships that could be construed as a potential conflict of interest.

\section{Conflicts of Interest}

The authors declare that they have no conflicts of interest.

\section{Acknowledgments}

The work was supported by the Jiangsu Provincial Key Research and Development Program (Grant No. BE2018757).

\section{References}

[1] S. J. Bakri, J. E. Thorne, A. C. Ho et al., "Safety and efficacy of anti-vascular endothelial growth factor therapies for neovascular age-related macular degeneration: a report by the American Academy of ophthalmology," Ophthalmology, vol. 126, no. 1, pp. 55-63, 2019.

[2] J. T. Handa, "How does the macula protect itself from oxidative stress?," Molecular Aspects of Medicine, vol. 33, no. 4, pp. 418-435, 2012.

[3] C. Roehlecke, U. Schumann, M. Ader et al., "Stress reaction in outer segments of photoreceptors after blue light irradiation," PLoS One, vol. 8, no. 9, article e71570, 2013.

[4] S. Datta, M. Cano, K. Ebrahimi, L. Wang, and J. T. Handa, "The impact of oxidative stress and inflammation on RPE degeneration in non- neovascular AMD," Progress in Retinal and Eye Research, vol. 60, pp. 201-218, 2017.

[5] T. Suzuki and M. Yamamoto, "Molecular basis of the Keap1Nrf2 system," Free Radical Biology and Medicine, vol. 88, Part B, pp. 93-100, 2015.

[6] S. H. Kim and J. W. Park, "Morin hydrate attenuates CSEinduced lipid accumulation, ER stress, and oxidative stress in RPE cells: implications for age-related macular degeneration," Free Radical Research, vol. 53, no. 8, pp. 865-874, 2019.

[7] B. Levine and G. Kroemer, "Autophagy in the pathogenesis of disease,” Cell, vol. 132, no. 1, pp. 27-42, 2008.
[8] P. Boya, L. Esteban-Martínez, A. Serrano-Puebla, R. GómezSintes, and B. Villarejo-Zori, "Autophagy in the eye: development, degeneration, and aging," Progress in Retinal and Eye Research, vol. 55, pp. 206-245, 2016.

[9] K. Kaarniranta, P. Tokarz, A. Koskela, J. Paterno, and J. Blasiak, "Autophagy regulates death of retinal pigment epithelium cells in age-related macular degeneration," Cell Biology and Toxicology, vol. 33, no. 2, pp. 113-128, 2017.

[10] E. Keeling, A. Lotery, D. Tumbarello, and J. Ratnayaka, "Impaired cargo clearance in the retinal pigment epithelium (RPE) underlies irreversible blinding diseases," Cells, vol. 7, no. 2, p. 16, 2018.

[11] I. Johansson, V. T. Monsen, K. Pettersen et al., "The marine n-3 PUFA DHA evokes cytoprotection against oxidative stress and protein misfolding by inducing autophagy and NFE2L2 in human retinal pigment epithelial cells," Autophagy, vol. 11, no. 9, pp. 1636-1651, 2015.

[12] Y. Mizunoe, M. Kobayashi, Y. Sudo et al., “Trehalose protects against oxidative stress by regulating the Keap1-Nrf2 and autophagy pathways," Redox Biology, vol. 15, pp. 115-124, 2018.

[13] L. Zhang, H. Wang, Y. Fan et al., "Fucoxanthin provides neuroprotection in models of traumatic brain injury via the Nrf2ARE and Nrf2-autophagy pathways," Scientific Reports, vol. 7, no. 1, article 46763, 2017.

[14] Y. Saito, Y. Kuse, Y. Inoue, S. Nakamura, H. Hara, and M. Shimazawa, "Transient acceleration of autophagic degradation by pharmacological Nrf2 activation is important for retinal pigment epithelium cell survival," Redox Biology, vol. 19, pp. 354-363, 2018.

[15] S. Dayalan Naidu, D. Dikovskaya, E. Gaurilcikaite et al., "Transcription factors NRF2 and HSF1 have opposing functions in autophagy," Scientific Reports, vol. 7, no. 1, article 11023, 2017.

[16] F. Li, F. Lang, H. Zhang et al., "Apigenin Alleviates EndotoxinInduced Myocardial Toxicity by Modulating Inflammation, Oxidative Stress, and Autophagy," Oxidative Medicine and Cellular Longevity, vol. 2017, Article ID 2302896, 10 pages, 2017.

[17] W. J. Hu, J. Liu, L. K. Zhong, and J. Wang, “Apigenin enhances the antitumor effects of cetuximab in nasopharyngeal carcinoma by inhibiting EGFR signaling," Biomedicine \& Pharmacotherapy, vol. 102, pp. 681-688, 2018.

[18] X. Xu, M. Li, W. Chen, H. Yu, Y. Yang, and L. Hang, “Apigenin attenuates oxidative injury in ARPE-19 cells thorough activation of Nrf2 pathway," Oxidative Medicine and Cellular Longevity, vol. 2016, Article ID 4378461, 9 pages, 2016.

[19] Y. Huang and W. G. Dai, "Fundamental aspects of solid dispersion technology for poorly soluble drugs," Acta Pharmaceutica Sinica B, vol. 4, no. 1, pp. 18-25, 2014.

[20] A. W. Khan, S. Kotta, S. H. Ansari, R. K. Sharma, and J. Ali, "Enhanced dissolution and bioavailability of grapefruit flavonoid naringenin by solid dispersion utilizing fourth generation carrier," Drug Development and Industrial Pharmacy, vol. 41, no. 5, pp. 772-779, 2014.

[21] P. Charbel Issa, A. R. Barnard, M. S. Singh et al., "Fundus autofluorescence in the Abca 4(-/-) mouse model of Stargardt disease -correlation with accumulation of A2E, retinal function, and histology," Investigative Ophthalmology \& Visual Science, vol. 54, no. 8, pp. 5602-5612, 2013.

[22] Q. Song, X. Sun, Q. Nie et al., "A novel method of multiparameter measurements for the mouse retina in vivo using optical coherence tomography," Experimental Eye Research, vol. 121 , pp. 66-73, 2014. 
[23] M. M. Edwards, D. S. McLeod, I. A. Bhutto, R. Grebe, M. Duffy, and G. A. Lutty, "Subretinal glial membranes in eyes with geographic atrophy," Investigative Ophthalmology \& Visual Science, vol. 58, no. 3, pp. 1352-1367, 2017.

[24] G. Landa, R. B. Rosen, J. Pilavas, and P. M. T. Garcia, "Drusen characteristics revealed by spectral-domain optical coherence tomography and their corresponding fundus autofluorescence appearance in dry age-related macular degeneration," Ophthalmic Research, vol. 47, no. 2, pp. 81-86, 2012.

[25] M. G. Nittala, H. Ruiz-Garcia, and S. R. Sadda, “Accuracy and reproducibility of automated drusen segmentation in eyes with non-neovascular age-related macular degeneration," Investigative Ophthalmology \& Visual Science, vol. 53, no. 13, pp. 8319-8324, 2012.

[26] F. C. Delori, C. K. Dorey, G. Staurenghi, O. Arend, D. G. Goger, and J. J. Weiter, "In vivo fluorescence of the ocular fundus exhibits retinal pigment epithelium lipofuscin characteristics," Investigative Ophthalmology \& Visual Science, vol. 36, no. 3, pp. 718-729, 1995.

[27] J. Lei, S. Balasubramanian, N. S. Abdelfattah, M. G. Nittala, and S. V. R. Sadda, "Proposal of a simple optical coherence tomography-based scoring system for progression of agerelated macular degeneration," Graefes Archive for Clinical and Experimental Ophthalmology, vol. 255, no. 8, pp. 15511558, 2017.

[28] J. M. Seddon, B. Rosner, R. D. Sperduto et al., "Dietary fat and risk for advanced age-related macular degeneration," Archives of Ophthalmology, vol. 119, no. 8, pp. 1191-1199, 2001.

[29] C. Su, Z. Liu, Y. Wang, Y. Wang, E. Song, and Y. Song, “The electrophilic character of quinones is essential for the suppression of Bach1," Toxicology, vol. 387, pp. 17-26, 2017.

[30] G. Bjørkøy, T. Lamark, A. Brech et al., "p 62/SQSTM1 forms protein aggregates degraded by autophagy and has a protective effect on huntingtin-induced cell death," Journal of Cell Biology, vol. 171, no. 4, pp. 603-614, 2005.

[31] Y. Kabeya, N. Mizushima, T. Ueno et al., "LC3, a mammalian homologue of yeast Apg8p, is localized in autophagosome membranes after processing," The EMBO Journal, vol. 19, no. 21, pp. 5720-5728, 2000.

[32] Q. M. Chen and A. J. Maltagliati, "Nrf2 at the heart of oxidative stress and cardiac protection," Physiological Genomics, vol. 50, no. 2, pp. 77-97, 2018.

[33] M. Pajares, N. Jiménez-Moreno, Á. J. García-Yagüe et al., "Transcription factor NFE2L2/NRF2 is a regulator of macroautophagy genes," Autophagy, vol. 12, no. 10, pp. 1902-1916, 2016.

[34] D. P. Frias, R. L. N. Gomes, K. Yoshizaki et al., "Nrf2 positively regulates autophagy antioxidant response in human bronchial epithelial cells exposed to diesel exhaust particles," Scientific Reports, vol. 10, no. 1, article 3704, 2020.

[35] L. F. Hernández-Zimbrón, R. Zamora-Alvarado, L. Ochoa-de la $\mathrm{Paz}$ et al., "Age-related macular degeneration: new paradigms for treatment and management of AMD," Oxidative Medicine and Cellular Longevity, vol. 2018, Article ID 8374647, 14 pages, 2018.

[36] R. K. Saini, K. R. R. Rengasamy, F. M. Mahomoodally, and Y. S. Keum, "Protective effects of lycopene in cancer, cardiovascular, and neurodegenerative diseases: an update on epidemiological and mechanistic perspectives," Pharmacological Research, vol. 155, article 104730, 2020.
[37] H. H. Dieguez, H. E. Romeo, A. Alaimo et al., "Oxidative stress damage circumscribed to the central temporal retinal pigment epithelium in early experimental non-exudative age-related macular degeneration," Free Radical Biology and Medicine, vol. 131, pp. 72-80, 2019.

[38] Age-Related Eye Disease Study Research Group, “A randomized, placebo-controlled, clinical trial of high-dose supplementation with vitamins $\mathrm{C}$ and $\mathrm{E}$ and beta carotene for age-related cataract and vision loss: AREDS report no. 9," Archives of Ophthalmology, vol. 119, no. 10, pp. 1439-1452, 2001.

[39] X. R. Xu, H. T. Yu, Y. Yang, L. Hang, X. W. Yang, and S. H. Ding, "Quercetin phospholipid complex significantly protects against oxidative injury in ARPE-19 cells associated with activation of Nrf2 pathway," European Journal of Pharmacology, vol. 770, pp. 1-8, 2016.

[40] B. Eisenhauer, S. Natoli, G. Liew, and V. Flood, "Lutein and zeaxanthin-food sources, bioavailability and dietary variety in age-related macular degeneration protection," Nutrients, vol. 9, no. 2, p. 120, 2017.

[41] C. Zhu, Y. Dong, H. Liu, H. Ren, and Z. Cui, "Hesperetin protects against $\mathrm{H} 2 \mathrm{O} 2$-triggered oxidative damage via upregulation of the Keap1-Nrf2/HO-1 signal pathway in ARPE-19 cells," Biomedicine \& Pharmacotherapy, vol. 88, pp. 124-133, 2017.

[42] P. Zeng, B. Liu, Q. Wang et al., “Apigenin Attenuates Atherogenesis through Inducing Macrophage Apoptosis via Inhibition of AKT Ser473 Phosphorylation and Downregulation of Plasminogen Activator Inhibitor-2," Oxidative Medicine and Cellular Longevity, vol. 2015, Article ID 379538, 12 pages, 2015.

[43] M. Kim, J. Jung, N. Y. Jeong, and H. J. Chung, "The natural plant flavonoid apigenin is a strong antioxidant that effectively delays peripheral neurodegenerative processes," Anatomical Science International, vol. 94, no. 4, pp. 285-294, 2019.

[44] J. Zhao, J. Yang, and Y. Xie, "Improvement strategies for the oral bioavailability of poorly water-soluble flavonoids: an overview," International Journal of Pharmaceutics, vol. 570, article 118642, 2019.

[45] Y. Shao, H. Yu, Y. Yang, M. Li, L. Hang, and X. Xu, "A solid dispersion of quercetin shows enhanced Nrf2 activation and protective effects against oxidative injury in a mouse model of dry age-related macular degeneration," Oxidative Medicine and Cellular Longevity, vol. 2019, Article ID 1479571, 12 pages, 2019.

[46] Z. Zhao, Y. Chen, J. Wang et al., "Age-related retinopathy in NRF2-deficient mice," PLoS One, vol. 6, no. 4, article e19456, 2011.

[47] K. T. Vu and J. D. Hulleman, "An inducible form of Nrf2 confers enhanced protection against acute oxidative stresses in RPE cells," Experimental Eye Research, vol. 164, pp. 31-36, 2017.

[48] X. Zhang, H. Bu, Y. Jiang et al., "The antidepressant effects of apigenin are associated with the promotion of autophagy via the mTOR/AMPK/ULK1 pathway," Molecular Medicine Reports, vol. 20, no. 3, pp. 2867-2874, 2019.

[49] X. Chen, H. Xu, X. Yu, X. Wang, X. Zhu, and X. Xu, "Apigenin inhibits in vitro and in vivo tumorigenesis in cisplatinresistant colon cancer cells by inducing autophagy, programmed cell death and targeting m-TOR/PI3K/Akt signalling pathway," Journal of BUON, vol. 24, no. 2, pp. 488-493, 2019. 
[50] L. Li, M. Li, S. Xu, H. Chen, X. Chen, and H. Gu, “Apigenin restores impairment of autophagy and downregulation of unfolded protein response regulatory proteins in keratinocytes exposed to ultraviolet B radiation," Journal of Photochemistry and Photobiology B, vol. 194, pp. 84-95, 2019.

[51] Z. Tang, B. Hu, F. Zang, J. Wang, X. Zhang, and H. Chen, "Nrf2 drives oxidative stress-induced autophagy in nucleus pulposus cells via a Keap1/Nrf2/p62 feedback loop to protect intervertebral disc from degeneration," Cell Death \& Disease, vol. 10, no. 7, p. 510, 2019.

[52] W. Liao, Z. Wang, Z. Fu et al., “p 62/SQSTM1 protects against cisplatin-induced oxidative stress in kidneys by mediating the cross talk between autophagy and the Keap 1-Nrf2 signalling pathway," Free Radical Research, vol. 53, no. 7, pp. 800-814, 2019.

[53] T. Jiang, B. Harder, M. Rojo de la Vega, P. K. Wong, E. Chapman, and D. D. Zhang, "p62 links autophagy and Nrf2 signaling," Free Radical Biology and Medicine, vol. 88, Part B, pp. 199-204, 2015. 